\title{
On the uniform Poincaré inequality
}

\author{
A. Boulkhemair ${ }^{1}$ and A. Chakib ${ }^{2}$ \\ ${ }^{1}$ Laboratoire de Mathématiques Jean Leray, \\ CNRS UMR6629/ Université de Nantes, \\ 2, rue de la Houssinière, BP 92208, 44322 Nantes, France. \\ E-mail: boulkhem@math.univ-nantes.fr \\ ${ }^{2}$ Département de Mathématiques Appliquées et Informatique \\ FST de Beni-Mellal, Université Cadi-Ayyad \\ B.P. 523, Beni-Mellal, Morocco. \\ E-mail: chakib@fstbm.ac.ma
}

\begin{abstract}
We give a proof of the Poincaré inequality in $W^{1, p}(\Omega)$ with a constant that is independent of $\Omega \in \mathcal{U}$, where $\mathcal{U}$ is a set of uniformly bounded and uniformly Lipschitz domains in $\mathbb{R}^{n}$. As a byproduct, we obtain the following : The first non vanishing eigenvalues $\lambda_{2}(\Omega)$ of the standard Neumann (variational) boundary value problem on $\Omega$ for the Laplace operator are bounded below by a positive constant if the domains $\Omega$ vary and remain uniformly bounded and uniformly Lipschitz regular.
\end{abstract}

\section{Introduction}

As it is well known, the Poincaré inequality is an important tool in the study of many problems of partial differential equations and numerical analysis. Others call it Poincaré-Friedrichs inequality. Recall that in the case of the usual Sobolev space $H^{1}(\Omega)=W^{1,2}(\Omega)$, it says the following: There exists a positive constant $C(\Omega)$ such that

$$
\|u\|_{L^{2}(\Omega)} \leq C(\Omega)\|\nabla u\|_{L^{2}(\Omega)}
$$

for all $u \in H^{1}(\Omega)$ such that

$$
\int_{\Omega} u d x=0 \text { or } u=0 \text { on some reasonable subset of } \bar{\Omega} .
$$

Here $\Omega$ is say a regular connected open set in $\mathbb{R}^{n}$. In this paper, we discuss the problem of the dependence of the constant $C(\Omega)$ on $\Omega$ without, however, looking for the best constant. In fact, in many applications, one needs to move the domain $\Omega$ and often needs to apply Poincaré inequality with a constant independent of the varying domains. This commonly happens, for example, in free boundary problems or shape optimization problems. See for example $[6,7]$. This also can happen when one simply needs to approximate the domain by more regular (or more "suitable") ones. See 
for example [8] where the authors studied a Robin problem on a Lipschitz domain and needed to apply a Poincaré (or Friedrichs) inequality whose constant is uniform with respect to the sequence of regular domains which approximates the Lipschitz one.

There are essentially two methods for proving the Poincaré inequality.

The first one, in fact the direct one, uses mainly the Taylor formula (or Newton formula) via the density of the regular functions in the Sobolev space. When one can perform it, it leads to an explicit constant $C(\Omega)$. For example, for a general domain $\Omega$ and under the condition $u=0$ on $\partial \Omega$, one obtains as $C(\Omega)$ an explicit function of the diameter of $\Omega$ or its measure. One can also

obtain an explicit $C(\Omega)$ when $\Omega$ is convex and the condition $\int_{\Omega} u d x=0$ is used. However, for a general geometry and under this null integral condition, it seems to be difficult to find an explicit reasonable constant $C(\Omega)$. To get an idea, see the recent attempt [12] where a more or less general geometry of $\Omega$ is used.

The other method of proof is an argument by contradiction using functional analysis. See for example [4]. The advantage of this method is that it allows to treat the case of a general domain with a minimal regularity and under any condition like that in (1). However, there is a trouble with it: It gives no information on the way the constant $C(\Omega)$ depends on $\Omega$, and so, when $\Omega$ moves we do not know what $C(\Omega)$ does in the general case.

For a while, we have been following the point of view that consists in seeking an explicit constant $C(\Omega)$. We did not succeed.

In this work, we solve our problem by considering a reasonable class of domains $\mathcal{U}$ for which we prove the Poincaré inequality with a constant independent of $\Omega \in \mathcal{U}$. Surprisingly, the method of proof is just the usual functional analysis argument by contradiction, in addition to few elementary techniques from shape optimization. The class $\mathcal{U}$ we consider is any set of domains that are uniformly bounded and have a uniform Lipschitz regularity.

Thanks are due to G. Carron and R. Souam for discussions on the geometry related to this subject.

\section{Notations and definitions}

We consider a fixed, open, bounded and regular subset $B$ of $\mathbb{R}^{n}$, which will contain all our domains. By a domain, we shall always mean an open connected subset of $\mathbb{R}^{n}$. Let us recall some definitions and notations which are needed below.

Let $\xi, y$ be vectors in $\mathbb{R}^{n}$ such that $|\xi|=1$ and $\varepsilon$ be a strictly positive number. The set defined by

$$
C(y, \xi, \varepsilon)=\left\{x \in \mathbb{R}^{n} ;|(x-y) \xi| \geq|x-y| \cos \varepsilon \text { and }|x-y|<\varepsilon\right\}
$$

is called the cone with vertex $y$, direction $\xi$ and angle to the vertex and height $\varepsilon$.

A domain $\Omega$ is said to have the $\varepsilon$-cone property if for all $x \in \partial \Omega$, there exists a direction $\xi$ and a positive real number $\varepsilon$ such that

$$
C(y, \xi, \varepsilon) \subset \Omega, \quad \text { for all } y \in B(x, \varepsilon) \cap \bar{\Omega}
$$

where $B(x, \varepsilon)$ is the open ball of center $x$ and radius $\varepsilon$ in $\mathbb{R}^{n}$.

Let us now recall some notions of convergence of sets. 
A sequence $\left(E_{k}\right)$ of measurable subsets of $\mathbb{R}^{n}$ is said to converge to a measurable subset $E$ of $\mathbb{R}^{n}$ in the sense of characteristic functions, and this is denoted by $E_{k} \stackrel{c h}{\longrightarrow} E$, if $\chi_{E_{k}} \rightarrow \chi_{E}$ in $L^{1}\left(\mathbb{R}^{n}\right)$, where $\chi_{A}$ denotes the characteristic function of the set $A$.

Let $K_{1}$ and $K_{2}$ be two compact subsets of $\mathbb{R}^{n}$, consider the usual distance from $x \in \mathbb{R}^{n}$ to $K_{i}$ $(i=1,2)$

$$
d\left(x, K_{1}\right)=\inf _{y \in K_{2}} d(x, y), \quad d\left(x, K_{2}\right)=\inf _{x \in K_{1}} d(x, y)
$$

and set

$$
\rho\left(K_{1}, K_{2}\right)=\sup _{x \in K_{1}} d\left(x, K_{2}\right) .
$$

By definition, the Hausdorff distance from $K_{1}$ to $K_{2}$, is the following non negative number

$$
d_{H}\left(K_{1}, K_{2}\right)=\max \left(\rho\left(K_{1}, K_{2}\right), \rho\left(K_{2}, K_{1}\right)\right)
$$

It is well known that this indeed defines a distance between compact subsets of $\mathbb{R}^{n}$.

Now, if $K_{j}, j \in \mathbb{N}$, and $K$ are compact subsets of $\mathbb{R}^{n}$, we shall write $K_{j} \stackrel{H}{\longrightarrow} K$ if $d_{H}\left(K_{j}, K\right) \rightarrow 0$ as $j \rightarrow \infty$.

Finally, if $\left(\Omega_{k}\right)_{k}$ is a sequence of open subsets of $B$ and $\Omega$ an open subset of $B$, we say that the sequence $\left(\Omega_{k}\right)_{k}$ converges to $\Omega$ in the Hausdorff (complement) sense and we denote it by $\Omega_{k} \stackrel{H^{c}}{\longrightarrow} \Omega$, if

$$
\bar{B} \backslash \Omega_{k} \stackrel{H}{\longrightarrow} \bar{B} \backslash \Omega \text {. }
$$

\section{Uniform Poincaré inequality}

Let us define the class $\mathcal{U}$ by

$$
\mathcal{U}=\{\Omega \subset B / \Omega \text { is open, connected and satisfies the } \varepsilon \text {-cone property }\} \text {. }
$$

Note that the elements of $\mathcal{U}$ are uniformly Lipschitz regular domains (see for example $[2,3,7,11]$ ). We shall need the following two lemmas.

Lemma 1 If $\left(\Omega_{k}\right)_{k}$ is a sequence in $\mathcal{U}$, then, there exists a subsequence of $\left(\Omega_{k}\right)_{k}$, denoted again $\left(\Omega_{k}\right)_{k}$, and an element $\Omega$ of $\mathcal{U}$ such that

$$
\Omega_{k} \stackrel{H^{c}}{\longrightarrow} \Omega, \quad \Omega_{k} \stackrel{c h}{\longrightarrow} \Omega \text { and } \bar{\Omega}_{k} \stackrel{H}{\longrightarrow} \bar{\Omega} .
$$

\section{Proof :}

Let $\left(\Omega_{k}\right)_{k}$ be a sequence in $\mathcal{U}$. It follows from a well known result in functional analysis related to shape optimization (see for example $[7,10,11]$ ), that there exists a subsequence denoted again $\left(\Omega_{k}\right)_{k}$ and an open subset $\Omega$ of $B$ having the $\varepsilon$-cone property such that

$$
\Omega_{k} \stackrel{H^{c}}{\longrightarrow} \Omega, \quad \Omega_{k} \stackrel{c h}{\longrightarrow} \Omega \text { and } \bar{\Omega}_{k} \stackrel{H}{\longrightarrow} \bar{\Omega} .
$$

According to Theorem 3.18 of [5], $\bar{\Omega}$ is connected. Since the domain $\Omega$ is Lipschitz regular, it is also connected. 
Lemma 2 If $E_{k}, k \in \mathbb{N}$, and $E$ are measurable subsets of $B, E_{k} \stackrel{\text { ch }}{\longrightarrow} E$ and $\left(f_{k}\right)_{k}$ is sequence of functions which converge to a function $f$ in $L^{1}(B)$, then we can extract subsequences denoted again $\left(E_{k}\right)_{k}$ and $\left(f_{k}\right)_{k}$ such that

$$
\lim _{k \longrightarrow \infty} \int_{E_{k}} f_{k} d x=\int_{E} f d x
$$

\section{Proof :}

Since $\left(E_{k}\right)_{k}$ is a convergent sequence to $E$ in the sense of characteristic functions, we can extract a subsequence denoted again $\left(E_{k}\right)_{k}$ such that $\chi_{E_{k}} \rightarrow \chi_{E}$ almost everywhere. Now, we have

$$
\begin{aligned}
\left|\int_{E_{k}} f_{k} d x-\int_{E} f d x\right| & =\left|\int_{B} \chi_{E_{k}} f_{k}-\chi_{E} f d x\right| \\
& \leq\left|\int_{B}\left(\chi_{E_{k}}-\chi_{E}\right) f d x\right|+\int_{B}\left|\chi_{E_{k}}\left(f_{k}-f\right)\right| d x \\
& \leq\left|\int_{B}\left(\chi_{E_{k}}-\chi_{E}\right) f d x\right|+\int_{B}\left|\left(f_{k}-f\right)\right| d x
\end{aligned}
$$

The lemma follows from the hypothesis and the Lebesgue convergence theorem.

Let us denote by $\mathcal{M}$ the set of measurable subsets of $B$. The main result of this paper is the following :

Theorem 1 Let $E: \mathcal{U} \longrightarrow \mathcal{M}$ be a map which is continuous in the sense that, if $\Omega_{k} \stackrel{\text { ch }}{\longrightarrow} \Omega$, then, $E\left(\Omega_{k}\right) \stackrel{\text { ch }}{\longrightarrow} E(\Omega)$. Assume also that $E(\Omega) \subset \Omega$ and $|E(\Omega)|>0$, for all $\Omega \in \mathcal{U}$. Then, there exists a constant $C>0$, such that

$$
\left\|u-\frac{1}{|E(\Omega)|} \int_{E(\Omega)} u d x\right\|_{p, \Omega} \leq C \sum_{i=1}^{n}\left\|\partial_{i} u\right\|_{p, \Omega}, \quad \forall u \in W^{1, p}(\Omega), \quad \forall \Omega \in \mathcal{U},
$$

where $\|\cdot\|_{p, \Omega}$ denotes the norm in $L^{p}(\Omega), p \geq 1$, and $|A|$ is the measure of the measurable set $A$.

\section{Proof :}

Assume that the result is false. Then, there exist sequences $\left(\Omega_{k}\right)_{k}$ in $\mathcal{U}$, and $\left(u_{k}\right)$ in $W^{1, p}\left(\Omega_{k}\right)$, such that, for all $k \geq 1$,

$$
\left\|u_{k}-\frac{1}{\left|E\left(\Omega_{k}\right)\right|} \int_{E\left(\Omega_{k}\right)} u_{k} d x\right\|_{p, \Omega}>k \sum_{i=1}^{n}\left\|\partial_{i} u_{k}\right\|_{p, \Omega_{k}} .
$$

If $\left(v_{k}\right)_{k}$ is the sequence defined by $v_{k}=u_{k}-\frac{1}{\left|E\left(\Omega_{k}\right)\right|} \int_{E\left(\Omega_{k}\right)} u_{k} d x$, we have

$$
\left\|v_{k}\right\|_{p, \Omega_{k}}>k \sum_{i=1}^{n}\left\|\partial_{i} v_{k}\right\|_{p, \Omega_{k}}, \quad \forall k \geq 1
$$

If $\left(w_{k}\right)_{k}$ is the sequence defined by $w_{k}=\frac{v_{k}}{\left\|v_{k}\right\|_{p, \Omega_{k}}}$, we have, for all $k \geq 1$,

$$
\left\|w_{k}\right\|_{p, \Omega_{k}}=1
$$

and

$$
\sum_{i=1}^{n}\left\|\partial_{i} w_{k}\right\|_{p, \Omega_{k}}<\frac{1}{k}
$$


Since the elements of $\mathcal{U}$ are uniformly Lipschitz regular, according to Chenais [3], we can construct a uniform extension operator $P_{\Omega}$ from $W^{1, p}(\Omega)$ to $W^{1, p}(B)$, for all $\Omega \in \mathcal{U}$, such that there exists a constant $M>0$ independent of $\Omega$ such that

$$
\left\|P_{\Omega} u\right\|_{W^{1, p}(B)} \leq M\|u\|_{W^{1, p}(\Omega)} .
$$

Set $\widetilde{w}_{k}=P_{\Omega_{k}} w_{k}$. For all $k \geq 1$, we have

$$
\begin{aligned}
\left\|\widetilde{w}_{k}\right\|_{W^{1, p}(B)} & \leq M\left\|w_{k}\right\|_{W^{1, p}(\Omega)}, \\
& \leq M\left(\left\|w_{k}\right\|_{p, \Omega}+\sum_{i=1}^{n}\left\|\partial_{i} w_{k}\right\|_{p, \Omega_{k}}\right) \\
& \leq M\left(1+\frac{1}{k}\right),
\end{aligned}
$$

hence,

$$
\left\|\widetilde{w}_{k}\right\|_{W^{1, p}(B)} \leq 2 M, \quad k \geq 1 .
$$

Since the sequence $\left(\widetilde{w}_{k}\right)_{k}$ is bounded in $W^{1, p}(B)$, there exists a subsequence denoted again $\left(\widetilde{w}_{k}\right)_{k}$ and an element $w \in W^{1, p}(B)(w \in B V(B)$ when $p=1)$ such that $\widetilde{w}_{k} \rightarrow w$ in $L^{p}(B)$. In particular, $\partial_{i} \widetilde{w}_{k}$ is convergent to $\partial_{i} w$ in $\mathcal{D}^{\prime}(B)$, for all $i(1 \leq i \leq n)$, where $\mathcal{D}^{\prime}(B)$ is the space of distributions in $B$. Now, according to Lemma 1 , the sequence $\left(\Omega_{k}\right)_{k}$ contains a subsequence denoted again $\left(\Omega_{k}\right)_{k}$ which converges to $\Omega \in \mathcal{U}$ in the Hausdorff sense. Let us show that $\partial_{i} w=0$ in $\Omega$, for all $i, 1 \leq i \leq n$. Indeed, let $\varphi \in \mathcal{D}(\Omega)$ (the space of $C^{\infty}$ functions with compact support in $\Omega$ ); it follows from the Hausdorff convergence of $\left(\Omega_{k}\right)_{k}$ to $\Omega$, that there exists $k_{0} \in \mathbb{N}$ such that $\varphi \in \mathcal{D}\left(\Omega_{k}\right), \forall k \geq k_{0}$. For all $i, 1 \leq i \leq n$, we have then

$$
\begin{aligned}
\left|\left\langle\partial_{i} w, \varphi\right\rangle\right| & =\left|\left\langle\partial_{i} w-\partial_{i} \widetilde{w}_{k}, \varphi\right\rangle+\left\langle\partial_{i} \widetilde{w}_{k}, \varphi\right\rangle\right| \\
& \leq\left|\left\langle\partial_{i} w-\partial_{i} \widetilde{w}_{k}, \varphi\right\rangle\right|+\left\|\partial_{i} \widetilde{w}_{k}\right\|_{p, \Omega_{k}}\|\varphi\|_{p^{\prime}, \Omega}, \quad \forall k \geq k_{0},
\end{aligned}
$$

where $p^{\prime}$ is such that $\frac{1}{p}+\frac{1}{p^{\prime}}=1$. Using (7) and passing to the limit, we obtain that

$$
\left\langle\partial_{i} w, \varphi\right\rangle=0, \quad \forall \varphi \in \mathcal{D}(\Omega) \text { and } \forall i, 1 \leq i \leq n .
$$

Thus, $\nabla w=0$ in $\Omega$. Since $\Omega$ is connected, it means that $w$ is constant in $\Omega$.

Now, we can assume that $\Omega_{k} \stackrel{c h}{\longrightarrow} \Omega$ as it follows from Lemma 1. Therefore, applying the continuity of $E$ and passing to the limit, we obtain that $E(\Omega) \subset \Omega$ up to a set of Lebesgue measure 0 . Let us show that $\int_{E(\Omega)} w d x=0$. Since $\widetilde{w}_{k} \rightarrow w$ in $L^{p}(B)$ and $E\left(\Omega_{k}\right) \stackrel{c h}{\longrightarrow} E(\Omega)$, it follows from Lemma 2 that

$$
\int_{E(\Omega)} w d x=\lim _{k \longrightarrow \infty} \int_{E\left(\Omega_{k}\right)} \widetilde{w}_{k} d x=0
$$

Since $w$ is constant in $\Omega$ and $E(\Omega)$ has a positive measure, we obtain that $w=0$ in $\Omega$. Finally, it follows from (6) and Lemma 2 that

$$
0=\int_{\Omega}|w|^{p} d x=\lim _{k \longrightarrow \infty} \int_{\Omega_{k}}\left|\widetilde{w}_{k}\right|^{p} d x=1
$$


which is a contradiction. This proves the theorem.

We state as a corollary, the following particular cases of the uniform Poincaré inequality of Theorem 1.

Corollary 1 (i) Under the assumptions of Theorem 1, there exists a constant $C>0$, such that

$$
\|u\|_{p, \Omega} \leq C \sum_{i=1}^{n}\left\|\partial_{i} u\right\|_{p, \Omega}, \quad \forall \Omega \in \mathcal{U} \text { and } \forall u \in W^{1, p}(\Omega), \text { such that } u=0 \text { in } E(\Omega) .
$$

(ii) If $E$ is a measurable subset of $B$ of positive measure, then, there exists a constant $C>0$ such that

$$
\|u\|_{p, \Omega} \leq C\left(\sum_{i=1}^{n}\left\|\partial_{i} u\right\|_{p, \Omega}+|E|^{\frac{1}{p}-1}\left|\int_{E} u d x\right|\right), \quad \forall u \in W^{1, p}(\Omega), \quad \forall \Omega \in \mathcal{U}, \quad E \subset \Omega .
$$

(iii) There exists a constant $C>0$ such that

$$
\|u\|_{p, \Omega} \leq C\left(\sum_{i=1}^{n}\left\|\partial_{i} u\right\|_{p, \Omega}+|\Omega|^{\frac{1}{p}-1}\left|\int_{\Omega} u d x\right|\right), \quad \forall u \in W^{1, p}(\Omega), \quad \forall \Omega \in \mathcal{U} .
$$

Note that parts $(i i)$ and $(i i i)$ of Corollary 1 correspond respectively to the cases $E(\Omega)=E$ and $E(\Omega)=\Omega$ of Theorem 1 .

Another consequence of Theorem 1 concerns the first non vanishing eigenvalue $\lambda_{2}(\Omega)$ of the standard Neumann (variational) boundary value problem on $\Omega$ for the Laplace operator. In fact, in the case $p=2$ and $E(\Omega)=\Omega$, it is well known that the best constant in the Poincaré inequality

$$
\left\|u-\frac{1}{|\Omega|} \int_{\Omega} u d x\right\|_{2, \Omega}^{2} \leq C \sum_{i=1}^{n}\left\|\partial_{i} u\right\|_{2, \Omega}^{2}
$$

is the inverse of $\lambda_{2}(\Omega)$, see Dautray-Lions [4], pp 925-927, for example. So, in this case, we can paraphrase Theorem 1 as follows :

Corollary 2 The eigenvalues $\lambda_{2}(\Omega)$ are bounded below by a positive constant if the domains $\Omega$ vary and remain uniformly bounded and uniformly Lipschitz regular.

Comment : Such a result may be of interest in geometry. Indeed, geometers usually seek lower bounds for the first eigenvalues of the standard Dirichlet and Neumann problems. See, for example, [13], [14]. The lower bound given by Corollary 2 may seem to be rough in comparison with what is known in geometry. Of course, this is because the geometric and regularity assumption, that is the cone assumption, made on the domains is very weak. It is a difficult problem to determine the constant as a function of $\Omega$ under such a general assumption.

Next, we try to go further and extend Theorem 1 to some smaller subsets $E(\Omega)$ of $B$. In fact, for simplicity, we state the result only for $E(\Omega)$ constant and equal to some hypersurface. One can certainly extend it to more general subsets by using the notion of capacity. 
Theorem 2 Let $\Gamma$ be a Lipschitz hypersurface in $\mathbb{R}^{n}$, possibly with a boundary, such that $\Gamma \subset B$ and $0<|\Gamma|<\infty,\left(|\Gamma|=\int_{\Gamma} d \sigma\right)$. Then, there exists a constant $C>0$, such that

$$
\left\|u-\frac{1}{|\Gamma|} \int_{\Gamma} u d \sigma\right\|_{p, \Omega} \leq C \sum_{i=1}^{n}\left\|\partial_{i} u\right\|_{p, \Omega}, \quad \forall u \in W^{1, p}(\Omega), p>1, \forall \Omega \in \mathcal{U}, \text { such that } \Gamma \subset \bar{\Omega} .
$$

\section{Proof :}

It follows the same scheme as that of Theorem 1, so we shall be brief. Assuming the statement false, there exist sequences $\left(\Omega_{k}\right)$ in $\mathcal{U}$ such that $\Gamma \subset \bar{\Omega}_{k}, \forall k \geq 1$, and $\left(w_{k}\right)$ in $W^{1, p}\left(\Omega_{k}\right)$ such that, for all $k \geq 1$,

$$
\left\|w_{k}\right\|_{p, \Omega_{k}}=1, \quad \sum_{i=1}^{n}\left\|\partial_{i} w_{k}\right\|_{p, \Omega_{k}}<\frac{1}{k} \text { and } \int_{\Gamma} w_{k} d \sigma=0
$$

As before, we define the sequence $\left(\widetilde{w}_{k}\right)$ which is bounded in $W^{1, p}(B)$ and we can assume it to converge weakly to $w \in W^{1, p}(B)$. Also, by a similar argument, one can show that $w$ is constant in $\Omega$, the limit of $\Omega_{k}$ for the Hausdorff convergence. Now, it follows from the compactness of the trace operator from $W^{1, p}(B)$ to $L^{p}(\Gamma)$ and the facts that $|\Gamma|<\infty$ and $\Gamma \subset \bar{\Omega}$, that $\int_{\Gamma} w d \sigma=$ $\lim _{k \rightarrow \infty} \int_{\Gamma} \widetilde{w}_{k} d \sigma=0$; so that $w=0$ in $\Omega$. Of course, this is in contradiction with the fact that

$$
\int_{\Omega}|w|^{p} d x=\lim _{k \rightarrow \infty} \int_{\Omega_{k}}\left|w_{k}\right|^{p} d x=1
$$

An immediate consequence of this theorem is the following

Corollary 3 Under the hypothesis of Theorem 2, there exists a constant $C>0$, such that

(i) $\quad\|u\|_{p, \Omega} \leq C\left(\sum_{i=1}^{n}\left\|\partial_{i} u\right\|_{p, \Omega}+|\Gamma|^{\frac{1}{p}-1}\left|\int_{\Gamma} u d x\right|\right), \quad \forall u \in W^{1, p}(\Omega), \quad \forall \Omega \in \mathcal{U}, \quad \Gamma \subset \bar{\Omega}$.

(ii)

$$
\|u\|_{p, \Omega} \leq C \sum_{i=1}^{n}\left\|\partial_{i} u\right\|_{p, \Omega} \quad \forall \Omega \in \mathcal{U}, \quad \Gamma \subset \bar{\Omega} \text { and } \forall u \in W^{1, p}(\Omega), \quad u=0 \text { on } \Gamma .
$$

\section{Remarks}

1. Let $P_{\Omega}: W^{1, p}(\Omega) \rightarrow W^{1, p}(B)$ be a uniform extension operator such as that of Chenais. Then, the uniform Poincaré inequality

$$
\|u\|_{p, \Omega} \leq C_{1} \sum_{i=1}^{n}\left\|\partial_{i} u\right\|_{p, \Omega}, \quad \forall \Omega \in \mathcal{U}, \quad \Gamma \subset \bar{\Omega} \text { and } \forall u \in W^{1, p}(\Omega), \quad u=0 \text { on } \Gamma,
$$

is equivalent to the following homogeneous estimate on the operator $P_{\Omega}$ :

$$
\sum_{i=1}^{n}\left\|\partial_{i} P_{\Omega} u\right\|_{p, B} \leq C_{2} \sum_{i=1}^{n}\left\|\partial_{i} u\right\|_{p, \Omega}, \quad \forall \Omega \in \mathcal{U}, \quad \Gamma \subset \bar{\Omega} \text { and } \forall u \in W^{1, p}(\Omega), \quad u=0 \text { on } \Gamma .
$$


In fact, this relies essentially on the Poincaré inequality applied to the fixed domain $B$.

This remark may be of interest mainly in the domain of shape optimization. It also gives another possible way of proof of the uniform Poincaré inequality.

2. The above results also hold when $p=\infty$, that is, in the case of Lipschitz functions. In fact, the proofs are rather elementary and one obtains an explicit constant $C(\Omega)$. For example, we can write

$$
\begin{aligned}
\mid u(x)- & \frac{1}{|E(\Omega)|} \int_{E(\Omega)} u(y) d y\left|\leq \frac{1}{|E(\Omega)|} \int_{E(\Omega)}\right| u(x)-u(y) \mid d y \\
& \leq \operatorname{diam}(\Omega) \sup _{x \neq y} \frac{|u(x)-u(y)|}{|x-y|} \leq \operatorname{diam}(B)\|\nabla u\|_{L^{\infty}(\Omega)},
\end{aligned}
$$

for all $u \in W^{1, \infty}(\Omega)$. One can even replace the average of $u$ on $E(\Omega)$ by the value of $u$ on some point $x_{\Omega} \in \Omega$, since the functions are continuous.

\section{References}

[1] Adams, R.A., Sobolev spaces, 65, Academic Press, New York, 1975.

[2] Chenais, D., "On the Existence of a Solution in a Domain Identification Problem", J. Mat. Annal. Appl., 52, (2) 189-289 (1975).

[3] Chenais, D., "Sur une famille de variétés à bord lipschitziennes. Application à un problème d'identification de domaine", Ann. Institut Fourier, 27, (4) 201-231 (1977).

[4] Dautray, R. and Lions, J.L., Analyse mathématique et calcul numérique pour les sciences et les techniques, Masson, Paris, 1988.

[5] Falconer, K. K., The geometry of fractal sets, Cambridge university press, 1985.

[6] Haslinger, J. and Neittaanmaki, P., Finite Element Approximtion for Optimal Shape Design. Theory and Applications, John and sons LTD, 1988.

[7] Henrot, A. and Pierre, M., Variation et optimisation de formes-Une analyse géométrique, Springer Series: Mathématiques et Applications, 48, 2005

[8] Lanzani, L. and Shen, Z., "On the Robin boundary condition for Laplace's equation in Lipschitz domains", Comm. Partial Differential Equations, 29 (1-2) 91-109 (2004).

[9] Neças, J., Les méthodes directes en théorie des équations elliptiques, Masson, Paris, 1967.

[10] Ly, I. and Seck, D., "Isoperimetric inequality for an interior free boundary problem with $p$ Laplacian operator", Electron. J. Differential Equations (109) 12 pp. (2004).

[11] Pironneau, O., Optimal Shape Design for Elliptic Systems, springer series in computational physics, springer-verlag, 1987.

[12] Weiying, Z. and He, Q. "On Friedrichs-Poincaré-type inequalities", Journal of Mathematical Analysis and Applications, 304 (2) 524-551 (2005).

[13] Gallot, S., "Minorations sur le $\lambda_{1}$ des variétés riemanniennes", Séminaire Bourbaki, 33e année, 1980/81, no 569 .

[14] Meyer, D., "Minoration de la première valeur propre non nulle du problème de Neumann sur les variétés riemanniennes à bord", Annales de l'institut Fourier, 36, no 2, (1986), p. 113-125. 